\title{
CLOSED ORBIT MEASUREMENT SYSTEM FOR THE BOOSTER SYNCHROTRON IN SRRC
}

\author{
K.H.Hu, Jenny Chen, K.T.Hsu, T. S. Ueng, S. Y. Hsu \\ Synchrotron Radiation Research Center \\ No 1, R\&D Road VI, Hsinchu Science-Based Industrial Park, Hsinchu, Taiwan, R.O.C
}

\section{Abstract}

The booster of SRRC is a $1.3 \mathrm{GeV}$ synchrotron with 10 $\mathrm{Hz}$ repetition rate. Electron beam from $50 \mathrm{MeV}$ accelerates to $1.3 \mathrm{GeV}$ within $50 \mathrm{msec}$. It serves as the injector for $1.3 \sim 1.5 \mathrm{GeV}$ storage ring of Taiwan Light Source (TLS). To reduce heat related problem in the machine and beamline optics, $1.5 \mathrm{GeV}$ full energy injection is scheduled in near future. The closed orbit measurement system of booster synchrotron consists of 23 set of BPMs, multiplexes, $500 \mathrm{MHz}$ receivers, timing control electronic and VME bus transient digitizer. The closed orbit is sampled every $150 \mu$ sec, 600 orbits acquired while the energy ramping. The closed-orbit is observed as function of energy. The orbit change due to imperfection of lattice and slow pulse magnet is easily observed. This system is expected to provide us abundant information to improve the operation efficiency, trouble shoot of booster.

\section{INTRODUCTION}

In the SRRC booster synchrotron, dipole and quadruple are drive by White circuit working in resonance excitation. To achieve high performance of the magnet system operation condition is crucial. Closedorbit measurement is helpful to optimization conditions of the magnet system, which includes excitation level, phasing of individual magnet family, and tracking between different family of magnet. The closed-orbit system to provide useful information of energy dependent in closed-orbit are related with tracking condition of magnet excitation, lattice error and correctors setting, optimized these injection and extraction elements condition. Booster energy is expected upgrade from $1.3 \mathrm{GeV}$ to $1.5 \mathrm{GeV}$ on October 1999. Major power supply will replace due to present equipment's have not enough rating to run in $1.5 \mathrm{GeV}$. At that time the beam closed-orbit can to become useful recommingling tool.

Several years ago, a simple standalone closed orbit measurement system has been setup [1]. In order to, understand more about the booster and its beam dynamic behavior, a new design was established. The new system will be integrated with the control system of the machine. An advanced graphical user interface will also be installed to aid machine physicists and operators.

\section{SYSTEM DESCRIPTIONS}

In the SRRC booster synchrotron, the beam position monitor (BPM) are locate in upstream and downstream of dipole magnets as shown in Fig. 1, except one location is reserved as synchrotron radiation diagnostic port (location 12 of BPM). There are 23 BPMs mounted around the ring totally for closed-orbit measurement.

The system use two stages $500 \mathrm{MHz}$ RF multiplexer to eliminate high cost matter, the multiplexes. The first stage RF multiplexes are similar with ESRF booster RF

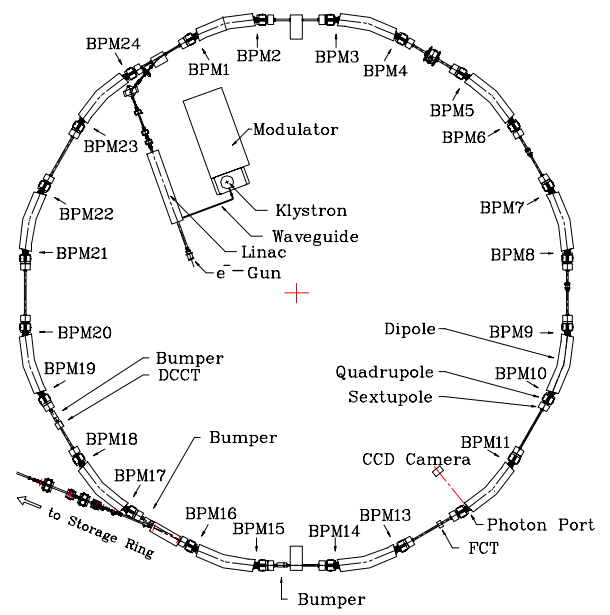

Figure 1: BPM layout in Booster synchrotron.

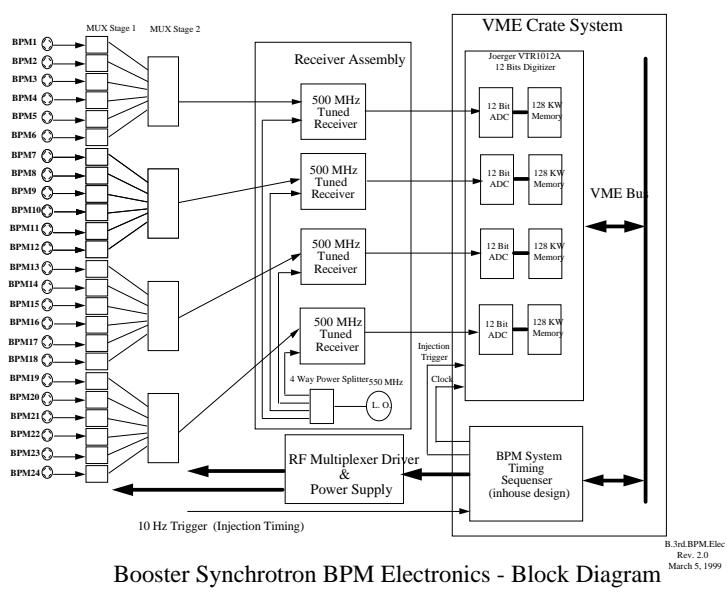

Figure 2: Closed Orbit measurement system.

MUX. The ESRF version work at $352 \mathrm{MHz}$ while SRRC work in $500 \mathrm{MHz}$. One BPM accompany with one 4-to1 RF multiplexer. The second are also 4-1 RF MUXs off-the-shelf products. All multiplexes driver and power supply are mounted on a distribution box.

The schematic diagram of the closed orbit 
measurement is shown in Fig.2. It uses 23 sets (BPM12 is dummy BPM) of electron beam position monitor. The BPMs divided into four-measurement group and parallel process to reduce the orbit acquisition cycle time. Each button takes $6 \mu \mathrm{sec}$ to acquire the signal from the electron beam. One processing channel handle 6 BPMs ( 24 buttons plus 1 reserved), But BPM12 is dummy. The acquisition time for each closed orbit need to $150 \mu \mathrm{sec}$. Four heterodyne receives are used in this system. The dynamic range of signal processing chain is about from $-60 \mathrm{dBm}$ to $0 \mathrm{dBm}$, which corresponds to beam current 0.1 to $10 \mathrm{~mA}$.

The timing sequence generator provides the clock for digitizer and the scan sequence of RF multiplexes. As the timing system receives enable and $10 \mathrm{~Hz}$ trigger signal, the data acquisition system and multiplexes system are immediately switched on. The signals picked up by the buttons of electrodes pass through the multiplexes and receiver to arrive the VME-based digitizer for further processing.

\section{SOFTWARE STRUCTURE}

The development of software for this closed orbit measurement system is divided into the low level data acquisition and the GUI presentation in the console. The law data acquisition software utilizes a VME based electronics system. It is responsible for setting parameters of the data acquisition module, calculating beam position from raw data, arranging the data acquisition, and uploading data to the console workstation. The function of software on workstation includes data request, user interface, and data presentation. The console display will show the beam position vs. energy, the closed orbit at different energy, the closed orbit difference between different energy, ...etc. Machine physicists and operator can easily to use the presentation to understand the condition of the booster.

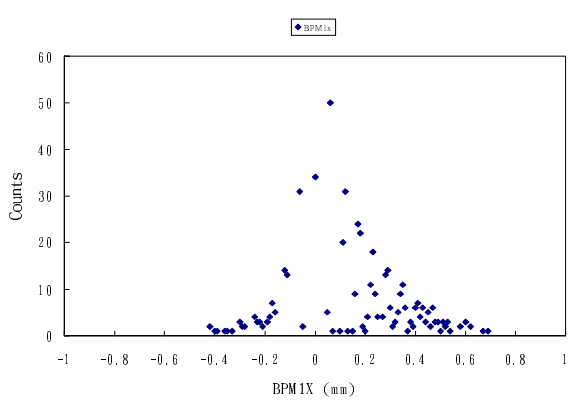

Figure 3: Histogram of beam position resolution, standard deviation $1 \sigma=200 \mu \mathrm{m}$.

\section{BEAM TESTS AND APPLICATION}

To verify performance of the system, various beam tested have been performed. Histogram of a typical beam position reading is shown in Fig. 3, the standard deviation is about $200 \mu \mathrm{m}$ which is the resolution of

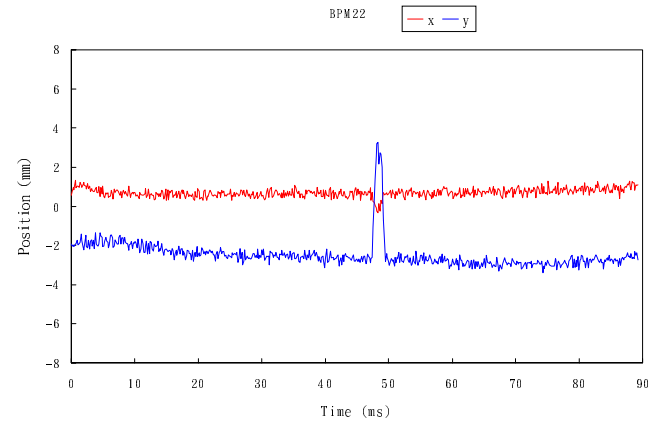

Figure 4: Typical beam position reading of BPM22 vs. time (energy).

BPM signal processing chain. That resolution can keep for the operating current range from 0.5 to $5 \mathrm{~mA}$ in multi-bunch operation mode.

The orbit displacement of electron beam in booster as a function of ramping energy is expected large, which is dependent on tracking of magnet excitation and error of lattice. Figure 4 shows the beam position varied as function of ramping time (energy). The electron beam is injected into the booster with energy of $50 \mathrm{MeV}$ at $0 \mathrm{msec}$. It increases gradually to $1.3 \mathrm{GeV}$ within $50 \mathrm{msec}$, then, decreases when passing $50 \mathrm{msec}$. Figure 4 shows that the beam position is changing at low energy side and approaches a steady state as beam energy rises higher enough. The spike shown in the beam position reading is due to the turn-on of extraction bumpers during the data taking.

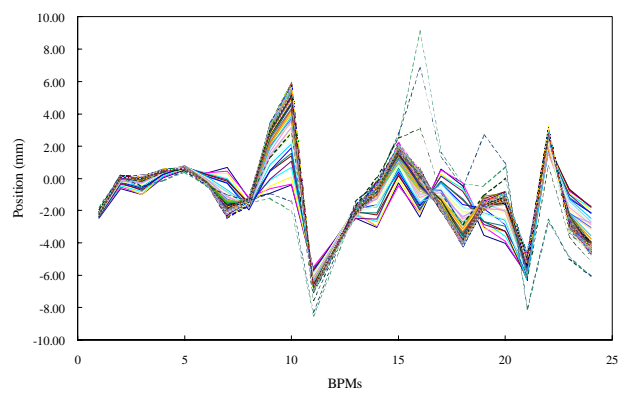

(a) Horizontal.

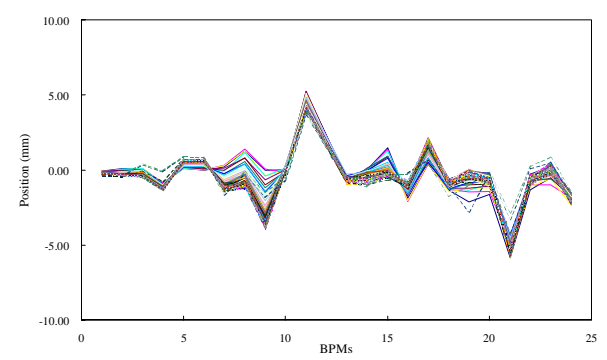

(b) Vertical.

Figure 5: $50 \mathrm{MeV} \sim 1.3 \mathrm{GeV}$ closed orbit variation. 


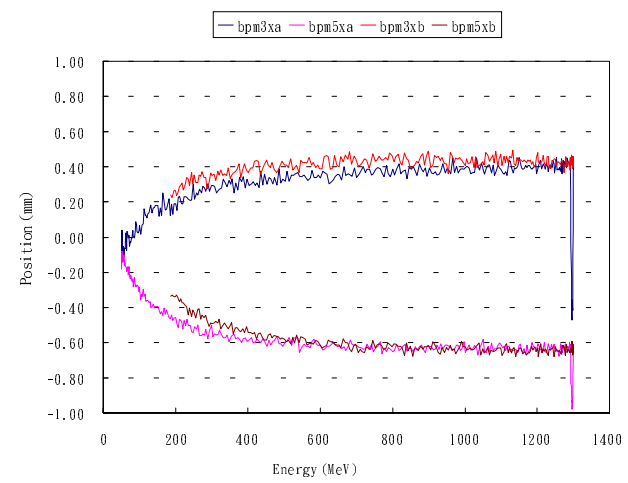

Figure 6: Beam position evolution of two BPMs vs. energy.

The energy dependence in closed orbit is related with tracking condition of magnet excitation, lattice error and corrector settings. To optimize these operating parameters, the closed orbit measurement at various beam energies is helpful. With an exaggerated tracking condition, the orbits displacement from 50 $\mathrm{MeV}$ to $1.3 \mathrm{GeV}$ at each BPM position are shown in Fig. 5. The actual displacement between these energies is small. The typical energy dependent position change of SRRC booster is shown in Fig. 6. In the figure, it is shown that the beam energy reaches $1.3 \mathrm{GeV}$ and decreases again, but the beam position does not follow the ramp up route. This phenomenon may be the result of tracking characteristics and hysteresis properties of magnet. As for which one is the major contributor, a further study should be performed.

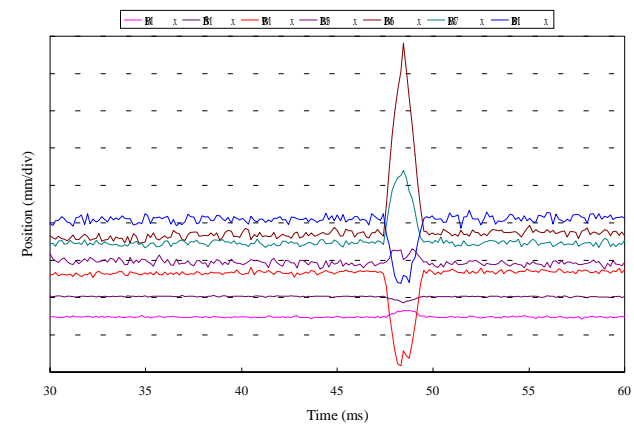

Figure 7: Beam position at serial BPMs, the bump occurs near $50 \mathrm{msec}$ is due to excitation bump turn on.

The extraction scheme of the booster synchrotron is to use one kicker, three extraction bumpers and one septum. Three extraction bumpers produce a $2 \mathrm{msec}$ half-sine bump to aid the extraction process. During the routine operation, the extraction condition seems not so critical to the beam extraction. However, the optimization of the extraction condition to improve the extraction efficiency and minimize the radiation loss is needed. It is clear that a non-closed bump was created between the first bumper and third bumper, seven BPMs (BPM 1,5,9,13,16,17,21) are selected for display purpose. BPM16 and BPM17 are located inside the bump. The bump occurs at about $50 \mathrm{msec}$ after the electron beam injected into the booster. All data have been added with different offset for display purpose. The optimization of bumper condition can be done easily by the closed orbit measurement system.

The condition of extraction septum is related to the extraction efficiency of the booster. During last several years, the short circuit of the extraction septum happened several occasions due to the electron beam hitting directly the structure of septum and the heat melted part of the structure. The beam position reading at upstream and downstream of extraction septum will be useful to assure the trajectory of stored beam and prevent electron beam from hitting the septum directly.

\section{SUMMARY}

We have set up a beam position acquisition system to acquire the closed orbit of SRRC booster synchrotron. The orbit acquisition time is $150 \mu \mathrm{sec}$. Its performance was evaluated by beam tests. The energydependent behavior of beam position was observed clearly. The bump produced by extraction bumpers was also studied $[2,3]$. The system will be useful to optimize the operation condition, such as tracking condition of magnet excitation, extraction bump, preventing electron beam from striking the septum, ...etc. A closed orbit measurement system will be also useful for the recommissioning of $1.5 \mathrm{GeV}$ booster synchrotron in the near future. Further study on the orbit response is planed [4]. The booster can also be operated at low currents $(<10 \mathrm{~mA})$, low energy $(<800 \mathrm{MeV})$, and short lifetime storage mode for various machine test in near future. At present, the rating of magnet power supplies, injection scheme and vacuum condition limit it. The closed orbit measurement system is expected to be useful for this mode of operation.

\section{REFERENCE}

[1] T. S. Ueng et al.," The Closed Orbit Measurement of SRRC Booster During Ramping “, Proceedings of the 1995 Particle Accelerator Conference, 2497-2499 (1995).

[2] R. Bakker, et al.," Fast Flexible BPM-System: Valuable Commissioning Tool for BESSY II ", Proceedings of the 6th European Particle Accelerator Conference, Stockholm, 1482 (1998).

[3] M. Abo-Bakr, et al.," Commissioning of the BESSY II Booster Synchrotron ", Proceedings of the 6th European Particle Accelerator Conference, Stockholm, 436 (1998).

[4] R. Bakker, et al.," Orbit Response Measurements in the Commissioning of the BESSY II Booster Synchrotron and Storage Ring ", Proceedings of the 6th European Particle Accelerator Conference, Stockholm, 900 (1998). 